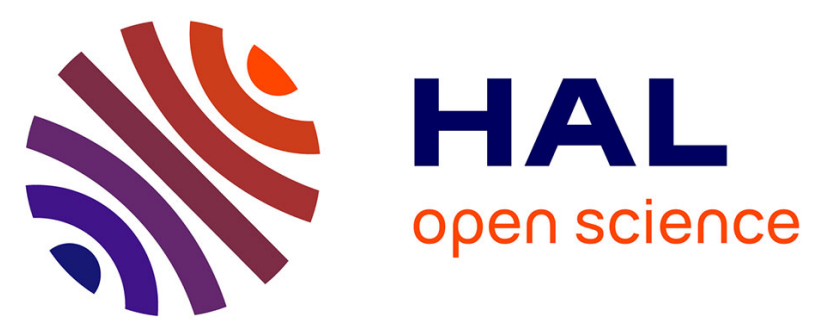

\title{
IRMPD spectroscopy and quantum chemistry calculations on mono- and bi-metallic complexes of acetylacetonate ligands with aluminum, iron and ruthenium ions
}

Nicolas Nieuwjaer, Ali Beydoun, Frédéric Lecomte, Bruno Manil, F

Cappelluti, Leonardo Guidoni, Debora Scuderi, Charles Desfrançois

\section{To cite this version:}

Nicolas Nieuwjaer, Ali Beydoun, Frédéric Lecomte, Bruno Manil, F Cappelluti, et al.. IRMPD spectroscopy and quantum chemistry calculations on mono- and bi-metallic complexes of acetylacetonate ligands with aluminum, iron and ruthenium ions. Journal of Chemical Physics, 2021, $10.1063 / 5.0031653$. hal-02860766v2

\section{HAL Id: hal-02860766 \\ https://hal.science/hal-02860766v2}

Submitted on 26 Nov 2020

HAL is a multi-disciplinary open access archive for the deposit and dissemination of scientific research documents, whether they are published or not. The documents may come from teaching and research institutions in France or abroad, or from public or private research centers.
L'archive ouverte pluridisciplinaire HAL, est destinée au dépôt et à la diffusion de documents scientifiques de niveau recherche, publiés ou non, émanant des établissements d'enseignement et de recherche français ou étrangers, des laboratoires publics ou privés. 


\title{
IRMPD spectroscopy and quantum chemistry calculations on mono- and bi-metallic complexes of acetylacetonate ligands with aluminum, iron and ruthenium ions
}

\author{
N. Nieuwjaer ${ }^{1}$, A. Beydoun ${ }^{1}$, F. Lecomte ${ }^{1}$, B. Manil' ${ }^{1}$, \\ F. Cappelluti ${ }^{3}$, L. Guidoni ${ }^{4}$, D. Scuderi ${ }^{2}$ and C. Desfrançois ${ }^{1 a)}$ \\ 1 Université Sorbonne Paris Nord, Lab. de Physique des Lasers, CNRS, 93430 Villetaneuse, France \\ 2 Université Paris Saclay, Institut de Chimie Physique, UMR 8000 CNRS, 91898 Orsay, France \\ 3 University of L'Aquila, Department of Information Engineering, Computer Science and Mathematics, 67000 \\ L'Aquila, Italy \\ 4 University of L'Aquila, Department of Physical and Chemical Sciences, 67000 L'Aquila, Italy
}

a) Author to whom correspondence should be addressed: charles.desfrancois@univ-paris13.fr

\begin{abstract}
Metal-ligand cluster ions are structurally characterized by means of gas-phase infrared multiple photon dissociation (IRMPD) spectroscopy. The mass-selected complexes consist of one or two metal cations $\mathrm{M}^{3+}(\mathrm{M}=\mathrm{Al}, \mathrm{Fe}$ or $\mathrm{Ru})$ and two to five anionic bidentate acetylacetonate ligands. Experimental IR spectra are compared with different density functional theory (DFT) calculations, namely PBE/TZVP, B3LYP/6-31G* and $M 06 / 6-31+\mathrm{G}^{* *}$. Frequency analysis was also performed at different levels, namely scaled static harmonic, unscaled static anharmonic or with ab initio molecular dynamics simulations at the PBE/TZVP level. All methods lead to simulated spectra that fit rather well with experimental data and the spectral red shifts of several main bands, in the $1200-1800 \mathrm{~cm}^{-}$ ${ }^{1}$ range, are sensitive to the strength of the metal-ligand interaction and to the spin state of the ion. Due to the rigidity of those complexes, first principles molecular dynamics calculations provide spectra similar to that produced by static calculations which are already able to catch the main spectral signatures using harmonic calculations at the B3LYP/6-31G* level.
\end{abstract}

Keywords: metal-ligand complexes; acetylacetonate; IRMPD spectroscopy; DFT calculations. 


\section{INTRODUCTION}

Many recent studies are devoted to functionalized metallic nanoparticles, for many applications: in Physics, due to their localized surface plasmon resonances [1]; in Chemistry, due to their specific catalytic properties [2]; in Biology, due to their optical [3] or magnetic [4] properties coupled to their potential for targeting and vectorization of bioactive molecules within living cells [5]. In solution, the characterization of the nanoparticle-ligand interface is often performed with NMR or IR methods, but data interpretations are somewhat difficult because of the relative lack of control of the stoichiometry of nanoparticles and of their ligand coverage [6].

Following a bottom-up approach, we here report on a work to study isolated metal-ligand clusters (few metal atoms, few ligands) that allow for a size selection with mass spectrometry techniques and for a structural characterization in the gas phase by IR spectroscopy. Their specific chemical/physical properties are very dependent on their size and structure and they are intermediate between those of the isolated metal and ligand and those of the liganded nanoparticles in solution [7,8]. Although many previous studies have been devoted to organometallic complexes in the gas phase $[9,10,11,12,13]$, most of them have concerned simple neutral $[14,15,16,17]$ or biomolecular ligands $[18,19,20,21,22]$. Up to our knowledge, apart from a recent study on holmium complexes [23], there is no other previous IR spectroscopy study of isolated complexes between metal ions and anionic acetylacetonate, especially when several metal ions are involved, although they are well known and characterized in their crystalline phase for a long time $[24,25,26]$ and although they are often used as precursors for the synthesis of various organometallic complexes and metal oxide nanoparticles [27].

We performed IR Multiple Photon Dissociation (IRMPD) spectroscopy experiments on isolated mass-selected metal-ligand ions whose results are compared to Density Functional Theory (DFT) calculations and vibrational spectra computed either at the scaled static harmonic or the unscaled static anharmonic levels or with ab initio molecular dynamics simulations. IRMPD spectroscopy is recognized as a powerful tool for structural characterization of very diverse molecular ions in the gas phase $[28,29,30]$ through the comparison of infrared signatures with model spectra that are predicted by DFT calculations. Agreement between experimental IRMPD and calculated DFT spectra is generally satisfying, in particular for band positions within tens of wavenumbers, although some larger differences are sometimes observed that can originate either from the experimental method 
(isomerization during the IRMPD process, red-shift due to the heating, IR intensities depending not only on the linear absorption) and more often from the calculation approximations (DFT method, empirical scaling of harmonic vibrational frequencies...).

In the present study, ions investigated involve light (Al), transition (Fe) and heavy $(\mathrm{Ru})$ metals and one of the simplest anionic bidentate ligands, acetylacetonate (Acac). Starting with small isolated species, our goal is to build some reliable methodology benchmarks for further spectroscopic experiments and DFT calculations concerning larger model systems for real liganded nanoparticles, as used by biochemists. Because of the relative rigidity of the present Acac anion ligand and its strong bidentate interactions with all metal ions, we will see that the low-energy structures of the cluster ions are straightforward in most cases and sometimes almost unique. The present data then constitute mainly a test of the validity of different approaches for simulating IR vibrational spectra for this class of metal-ligand cluster ions.

\section{EXPERIMENTAL AND THEORETICAL METHODS}

Solid $\mathrm{Al}(\mathrm{III})$ and Fe(III) acetylacetonate were purchased from a commercial source (Sigma-Aldrich, purity $>97 \%$ ) and used without further purification in 1:1 solutions of water and methanol at concentrations of 10 to $100 \mu \mathrm{M}$. Solid $\mathrm{Ru}(\mathrm{III})$ acetylacetonate was synthesized in the group of $\mathrm{J}$. Crassous ((Rennes Institute of Chemical Sciences) and used in the same conditions.

IRMPD experiments have been performed at the free electron laser (FEL) of the Centre Laser Infrarouge d'Orsay (CLIO) facility, in the $400-1900 \mathrm{~cm}^{-1}$ spectral range. Ion spectra were recorded using the available $7 \mathrm{~T}$ hybrid FT-ICR mass spectrometer (APEX-Qe Bruker) coupled to the CLIO FEL beamline. This experimental setup has been previously described in detail $[31,32]$. Briefly, ions in solution were delivered in the gas-phase by an electrospray ionization (ESI) source with typical extraction voltage of $4 \mathrm{kV}$ and desolvation temperature of $150-200{ }^{\circ} \mathrm{C}$. Ions were accumulated, premass-selected and thermalized in a quadrupole-hexapole interface and then pulse extracted towards the FT-ICR cell maintained under high vacuum $\left(<10^{-9} \mathrm{mbar}\right)$ and at room temperature. Massselected ions were stored and irradiated for 0.5 to $2 \mathrm{~s}$ with the IR-FEL light in the frequency range of interest. Depending on the electron energy used (35-45 MeV) for covering a given spectral range, the average CLIO laser power is recorded and lies in between 0.5 and $1.2 \mathrm{~W}$. Its frequency resolution, about or lower than $1 \%$, and the spectral rotational broadening of ions at $300 \mathrm{~K}$, lead to 
an overall linewidth of about $10 \mathrm{~cm}^{-1}$. If necessary for revealing weak vibrational bands, and in order to increase fragmentation for strongly bound systems, ions were also submitted to an auxiliary broadband $\mathrm{CO}_{2}$ laser synchronized with CLIO-FEL (Universal Laser System, $10 \mathrm{~W}$, cw operation centered at $\lambda=10.6 \mu \mathrm{m}$ ), at the beginning of the FEL irradiation period and for times varying between one and few tens of ms. The $\mathrm{CO}_{2}$ laser pulse length was adjusted for each experiment to avoid photodissociation of the molecule by the $\mathrm{CO}_{2}$ laser alone while promoting the fragmentation in the presence of the CLIO laser.

When the IR-FEL light is in resonance with an active vibrational mode of the mass-selected ions, multiple and sequential events of photon absorption and intramolecular vibrational energy redistribution (IVR) ultimately activate their fragmentation [33]. At each spectral wavenumber, typically five mass spectra were recorded and accumulated in order to compare the integrated number of precursor (Ip) and fragment (If) ions as a function of the laser frequency. An IRMPD spectrum is then obtained by recording the photofragmentation yield, $R=-\ln [\operatorname{lp} /(\operatorname{Ip}+\Sigma \mid \mathrm{f})]$, as a function of the wavenumber of the IR-FEL radiation.

All static DFT calculations have been performed using the Gaussian 16 package [34] on the HPC platform MAGI of Université Sorbonne Paris Nord. Full geometry optimizations have been performed first at the UB3LYP/6-31G* basic level $[35,36,37,38]$, for different spin multiplicity and starting from different trial structures inspired by those of the crystalline phase. For the Ru atom, we used the Def2TZVP [39] or the LanL2DZ [40] basis set which includes an effective core potential for heavy transition metals. In order to test the validity of the B3LYP functional and the modest $6-31 G^{*}$ basis set, in particular with respect to the spin ground state, we also performed calculations with the M06 functional [41], which is supposed to give better energies for transition metal chemistry [42,43], at the $\mathrm{M} 06 / 6-31+\mathrm{G}^{* *}$ level. In order to ascertain which spin state is the lowest one, we always performed calculations with different spin multiplicity values around that of lowest total electronic energy. For $\mathrm{Al}(\mathrm{III})$, there is no electron in d orbital so that the spin must be zero but for both $\mathrm{Fe}(\mathrm{III})$ and $R u(I I I)$, there are five $d$ electrons and the resulting spin can thus be in between $1 / 2$ and $5 / 2$, corresponding to one to five unpaired electrons. In the species studied, it appears that all complexes with $\mathrm{Fe}(\mathrm{III})$ always display high spin states (5/2), while $\mathrm{Ru}(\mathrm{III})$ complexes always correspond to low spin states (1/2). Note that we also performed some ab initio calculations at the UMP2/6-31+G** [44] 
level which were fully coherent with the DFT energetics and did not bring any interesting added value for IR spectra, so they are not reported here.

Frequency analysis was performed at the same levels of theory either in the harmonic or the anharmonic approximation as implemented in Gaussian 16 [34]. For direct comparison with the measured IRMPD spectra, the calculated vibrational line spectra were convoluted using a Lorentzian line shape function with a full width at half-maximum value of $10 \mathrm{~cm}^{-1}$. For anharmonic results, no frequency scaling was applied while for harmonic calculations, a frequency scaling factor was determined to fit as best as possible the band positions of the calculated anharmonic spectra. As discussed below, in most cases, a standard scaling factor of 0.975 was suitable both for B3LYP and M06 calculations.

DFT-MD simulations were also performed through Born-Oppenheimer Molecular Dynamics as implemented in the CP2K package [45] using Galileo and Marconi HPC platforms from Cineca. The cubic box size was set as twice the largest dimension of the complex, therefore around 20-22 A, depending on the system. IR spectra were obtained by calculating the power spectra of the dipole autocorrelation functions, evaluated using Wannier centers [46] for electron localization. Molecular dynamics simulations in the NVE ensemble have been performed on single molecular ions at the PBE/TZVP $[47,48]$ level with GTH-SR-MOLOPT pseudopotentials [49], using a plane wave cutoff of 460 Rydberg and Grimme's D3 correction [50] for dispersion interactions (with a 12.5 Angstrom cutoff). Prior to the production simulation time of 20 ps, a 2 ps long NVT equilibration at $298 \mathrm{~K}$ has been performed and spectra have been obtained by applying a Lorentzian smoothing with a width of $5 \mathrm{~cm}^{-1}$ but with no frequency scaling. B3LYP-MD simulations have not been conducted due to the too large computational effort required and, for the same reason, we did not perform DFT-MD calculations involving Ru complexes. For comparison with those dynamic calculations and the other static calculations with B3LYP and M06 functionals, static harmonic or anharmonic frequency calculations have also been performed at the same PBE/TZVP level using CP2K with the same computational setup. 


\section{RESULTS AND DISCUSSION}

\section{A. Mass spectrometry}

In the condensed phase, metal-acetylacetonate (M-Acac) complexes are known to form very stable $\mathrm{M}^{3+}\left(\mathrm{Acac}^{-}\right)_{3}$ octahedral neutral species with well-known crystalline structures $[24,51,52]$. High resolution mass spectrometry allows us to resolve the isotopic patterns of the observed ions. Upon $\mathrm{ESI}$ in the positive mode, the main observed ions are the singly charged $\left[\mathrm{M}^{3+}\left(\mathrm{Acac}^{-}\right)_{2}(\mathrm{AcacH})\right]^{1+}$ species that are the cationic counterparts of those neutral in solution, in which one Acac anion has captured one proton and becomes a neutral acetylacetone $(\mathrm{AcacH})$ molecule that can be easily lost by collision-induced dissociation (CID). For Al and Fe (see Fig. 1 and 2 in the supplementary material), we also observed rather high intensities of $\left[\mathrm{M}^{3+}\left(\mathrm{Acac}^{-}\right)_{2}\right]^{1+}$ ions, which can originate from the loss of a neutral $\mathrm{AcacH}$, but also intense $\left[\mathrm{M}^{3+}{ }_{2}\left(\mathrm{Acac}^{-}\right)_{5}\right]^{1+}$ precursor ions, whose main $\mathrm{CID}$ fragments are $\left[\mathrm{M}^{3+}\left(\mathrm{Acac}^{-}\right)_{2}\right]^{1+}$ by loss of stable neutrals $\mathrm{M}^{3+}\left(\mathrm{Acac}^{-}\right)_{3}$. In the case of $\mathrm{Al}$, we also see intense ions at $\mathrm{m} / \mathrm{z} 467.14$, which isotopic mass and profile corresponds very well to $\left[\mathrm{Al}^{3+}{ }_{2}\left(\mathrm{Acac}^{-}\right.\right.$ $\left.{ }_{4} \mathrm{OH}^{-}\right]^{1+}$ ions, where one acetylacetonate is replaced by one hydroxyl anion (see Fig. 3 in the supplementary material). For $\mathrm{Ru}$ complexes, we also observe $\left[\mathrm{Ru}^{3+}\left(\mathrm{Acac}^{-}\right)_{2}(\mathrm{AcacH})\right]^{1+}$ ions but surprisingly no $\left[\mathrm{Ru}^{3+}\left(\mathrm{Acac}^{-}\right)_{2}\right]^{1+}$ ions, while the main isotopic pattern corresponds to $\left[\mathrm{Ru}^{2+}\left(\mathrm{Acac}^{-}\right.\right.$ )$\left._{1}(\mathrm{AcacH})_{3}\right]^{1+}$ ions (see Fig. 4 and 5 in the supplementary material). We also note that for $\mathrm{Ru}(\mathrm{IV})$ we see weak signals corresponding to $\left[\mathrm{Ru}^{4+}\left(\mathrm{Acac}^{-}\right)_{1}(\mathrm{AcacH})_{3}\right]^{3+}$ ions but no signal which could corresponds to $\left[\mathrm{Ru}^{4+}\left(\mathrm{Acac}^{-}\right)_{2}\right]^{2+}$. It is thus concluded that $\mathrm{Ru}$ hardly forms stable complexes with only two Acac ligands but complexes only with three ligands for $\mathrm{Ru}(\mathrm{III})$ and four ligands for $\mathrm{Ru}(\mathrm{II})$ or $\mathrm{Ru}(\mathrm{IV})$, at least from ESI in our experimental conditions. In this report, we will however restrict our results and discussion to $\mathrm{M}^{3+}$ complexes.

\section{B. $\left[M^{3+}\left(\mathrm{Acac}^{-}\right)_{2}(\mathrm{AcacH})\right]^{1+}, \mathrm{M}=\mathrm{Al}, \mathrm{Fe}, \mathrm{Ru}$}

These species contain three ligands around the metal ion in the oxidation state (III) and are expected to share similar IR spectra corresponding to similar octahedral structures, like the neutral species in the condensed phase, in which the three bidentate ligands form six bonds with the metal ion. As expected, their fragmentation by CID or IRMPD occurs mainly through the loss of the neutral AcacH molecule. Figure 1 displays the experimental IRMPD spectra for the three metals studied. As expected, they share several common features, although the Ru spectrum is red-shifted by $50-70$ 
$\mathrm{cm}^{-1}$ as compared to $\mathrm{Al}$ and Fe spectra that are close together, indicating that the Ru-Acac interactions should be stronger than the similar ones with $\mathrm{Al}$ and $\mathrm{Fe}$, as discussed below.

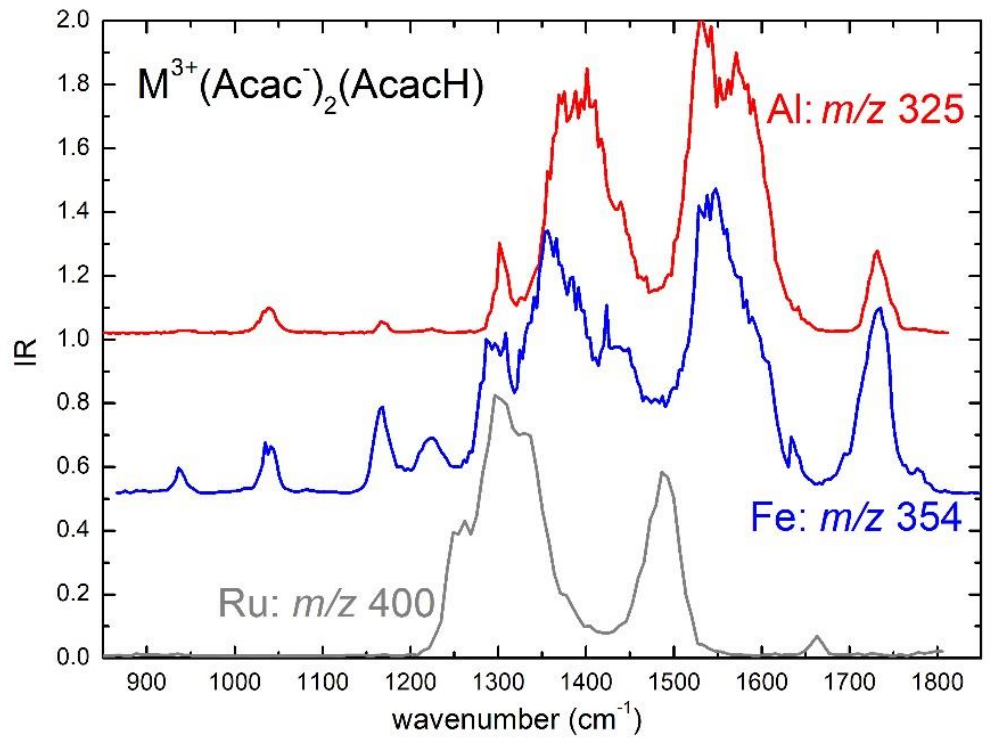

FIG. 1: IRMPD spectra of the three metal-Acac cations studied. In all experiments, no pre-heating $\mathrm{CO}_{2}$ laser and reduced IR-FEL irradiation times were used $(0.25 \mathrm{~s}$ for $\mathrm{Al}, 0.5 \mathrm{~s}$ for $\mathrm{Fe}$ and $2 \mathrm{~s}$ for $\mathrm{Ru})$. As in the following spectra, experimental IR intensities are photofragmentation yields as defined above and scaled in order to be of comparable heights for the main vibrational band.

Figure 2 displays the comparison between experimental and calculated IR spectra for AI and Fe.
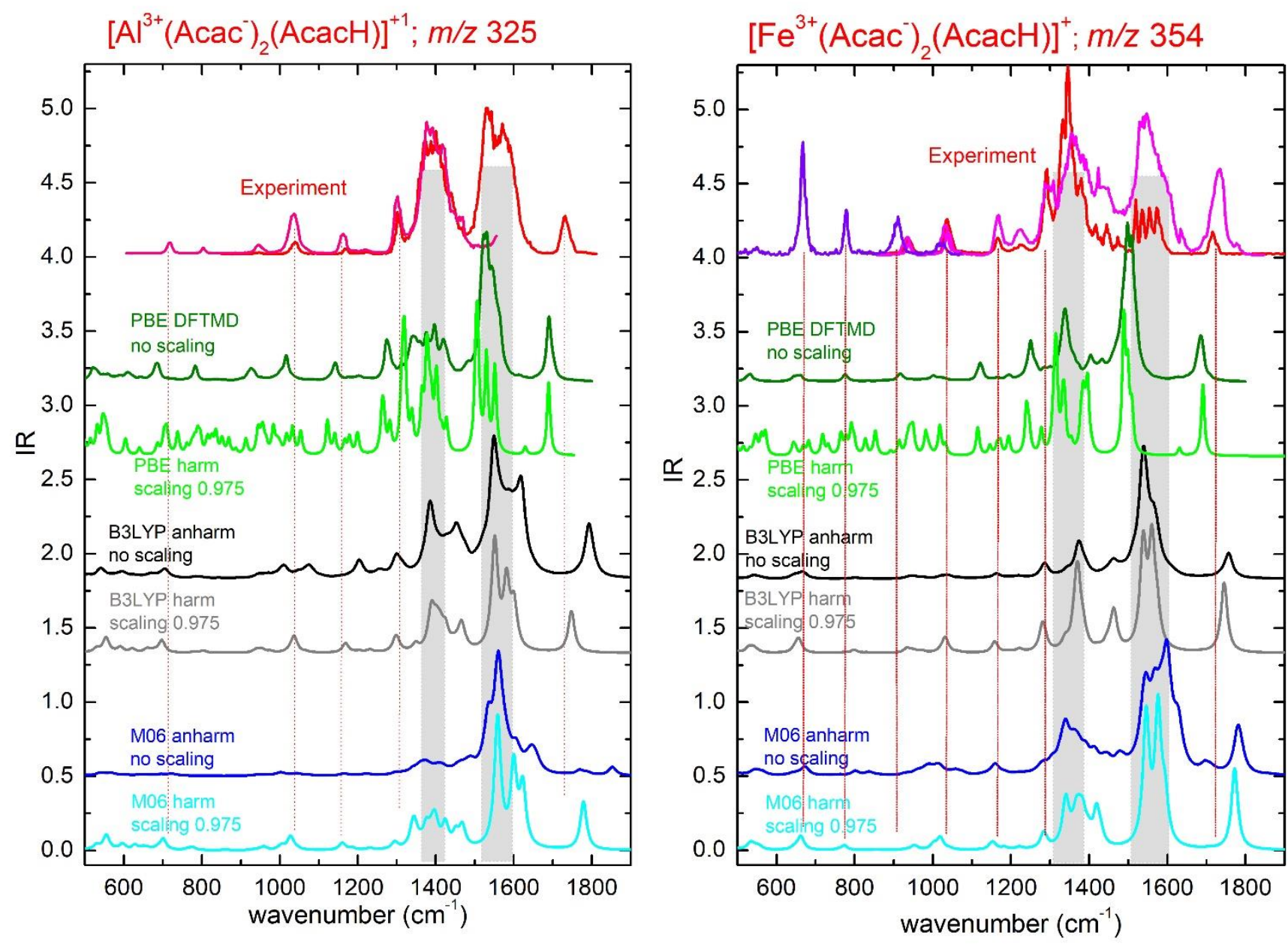

FIG. 2: Experimental IRMPD spectra for $\left[\mathrm{Al}^{3+}\left(\mathrm{Acac}^{-}\right)_{2}(\mathrm{AcacH})\right]^{1+}$ and $\left[\mathrm{Fe}^{3+}\left(\mathrm{Acac}^{-}\right)_{2}(\mathrm{AcacH})\right]^{1+}$ (top curves) as compared to calculated scaled harmonic (light colors) and unscaled anharmonic (dark colors) IR spectra at the M06/6-31+G** (blue) and B3LYP/6-31G* (black), together with PBE/TZVP calculations performed at the scaled harmonic static level (light green) and at the unscaled dynamic level through DFT-MD simulations (dark green). The two main bands around $1300-1400 \mathrm{~cm}^{-1}$ and $1500-1600 \mathrm{~cm}^{-1}$ are grayed out and other main experimental peaks are marked with dotted lines in order to guide eyes. 
Experimental data display the two or three runs that have been performed in different frequency ranges and at different IR-FEL laser powers so that bands and lines appear with various relative intensities. Calculated IR intensities are also very different from one method to another and all IR intensities are thus scaled so that the main peak intensity is normalized to about 1 in this figure. All calculated spectra reproduce quite well the position of the two main bands that correspond to different $\mathrm{C}=\mathrm{O}$ and $\mathrm{C}-\mathrm{C}=\mathrm{O}$ symmetric and asymmetric stretches of neutral or anionic Acac. The only discrepancy concerns the PBE calculations for iron complexes with the band at $1500-1600 \mathrm{~cm}^{-1}$ that is too red-shifted as compared to experiment. It seems that this functional tends to overestimate the interaction between the negatively charge oxygen in Acac $^{-}$and the aluminum or iron metal ion.

Moreover, there is also a good and even outstanding agreement for the lines of lower intensities and frequencies that appears at 1302, 1163, 1035, very small 946 and 804 , and $717 \mathrm{~cm}^{-1}$ for $\mathrm{Al}$ and 1293 , 1167, 1036, 930, 792 and $677 \mathrm{~cm}^{-1}$ for Fe. Most of these modes can be assigned to either anion Acac $^{-}$and neutral AcacH species that are respectively more and less red-shifted in the complex. For instance, the line at $1163 \mathrm{~cm}^{-1}$ for $\mathrm{Al}$ (or $1167 \mathrm{~cm}^{-1}$ for Fe) corresponds to a red-shifted asymmetric stretching mode involving the five carbon atoms that is calculated in neutral $\mathrm{AcacH}$ at $1200 \mathrm{~cm}^{-1}$.

On the other hand, the line at high frequency, experimentally found at $1733 \mathrm{~cm}^{-1}$ for both $\mathrm{Al}$ and Fe, is not so well reproduced by none of the methods used. It is calculated either at too low frequency with PBE or at too high frequencies with B3LYP and M06. This mode corresponds to the symmetric two $\mathrm{C}=\mathrm{O}$ stretches of neutral $\mathrm{AcacH}$ that is calculated at $1755 \mathrm{~cm}^{-1}$ for the isolated neutral. This again indicates that PBE probably overestimates the interactions between metallic cations and neutral oxygen atoms, while B3LYP and M06 underestimate it.

More quantitatively, we have calculated the root-mean-square deviations (RMSD) between the present types of frequency calculations and the experimental values, for the main nine lines and bands that are outlined in Fig. 2 (see tables I and II in the supplementary material). From these values, it clearly appears that static B3LYP scaled harmonic frequency calculations perform much better $\left(\mathrm{RMSD}=15.3 \mathrm{~cm}^{-1}\right.$ for $\mathrm{Al}$ and $11.1 \mathrm{~cm}^{-1}$ for $\left.\mathrm{Fe}\right)$ than other methods $\left(\mathrm{RMSD} \sim 22 \mathrm{~cm}^{-1}\right.$ with M06 and around $30 \mathrm{~cm}^{-1}$ with PBE) and that unscaled static anharmonic frequency calculation results are always worse than scaled harmonic ones (with B3LYP, RMSD $=28.8 \mathrm{~cm}^{-1}$ for Al and $13.2 \mathrm{~cm}^{-1}$ for Fe). Dynamical DFT-MD frequency calculations do not perform better than static ones (RMSD = 
$24.2 \mathrm{~cm}^{-1}$ for $\mathrm{Al}$ and $34.7 \mathrm{~cm}^{-1}$ for Fe) but we observed a systematic red shift, of the order of $25-30$ wavenumbers, that accounts for the main part of those relatively bad RMSD and, when this systematic error is empirically compensated, the RMSD are improved a lot and become the lowest ones $\left(\mathrm{RMSD}^{*}=8.6 \mathrm{~cm}^{-1}\right.$ for $\mathrm{Al}$ and $15.6 \mathrm{~cm}^{-1}$ for $\left.\mathrm{Fe}\right)$. We could not find in the literature other examples of this compensation, that can probably be explained as a consequence of the excessive loosening of bonds (and reduction of vibrational frequencies) produced by GGA functionals [53].

Figure 3 displays the same experimental and calculated IR spectra for $\mathrm{Ru}$, together with the picture of the calculated equilibrium structure. From the two experimental runs displayed, it is clearly seen that pre-heating helps to enhance low intensity lines, particularly the one at higher frequency, but also leads to a widening of all lines, including the two main bands that appear here around 1320 and $1490 \mathrm{~cm}^{-1}$. For both M06 and B3LYP calculations, these two bands are not as red-shifted, with respect to the previous $\mathrm{Al}$ and Fe complexes, as compared to the experiments results: $-20 \mathrm{~cm}^{-1}$ instead of $50-70 \mathrm{~cm}^{-1}$. In that case, PBE functional is performing better, with a calculated red-shift of about $50 \mathrm{~cm}^{-1}$.

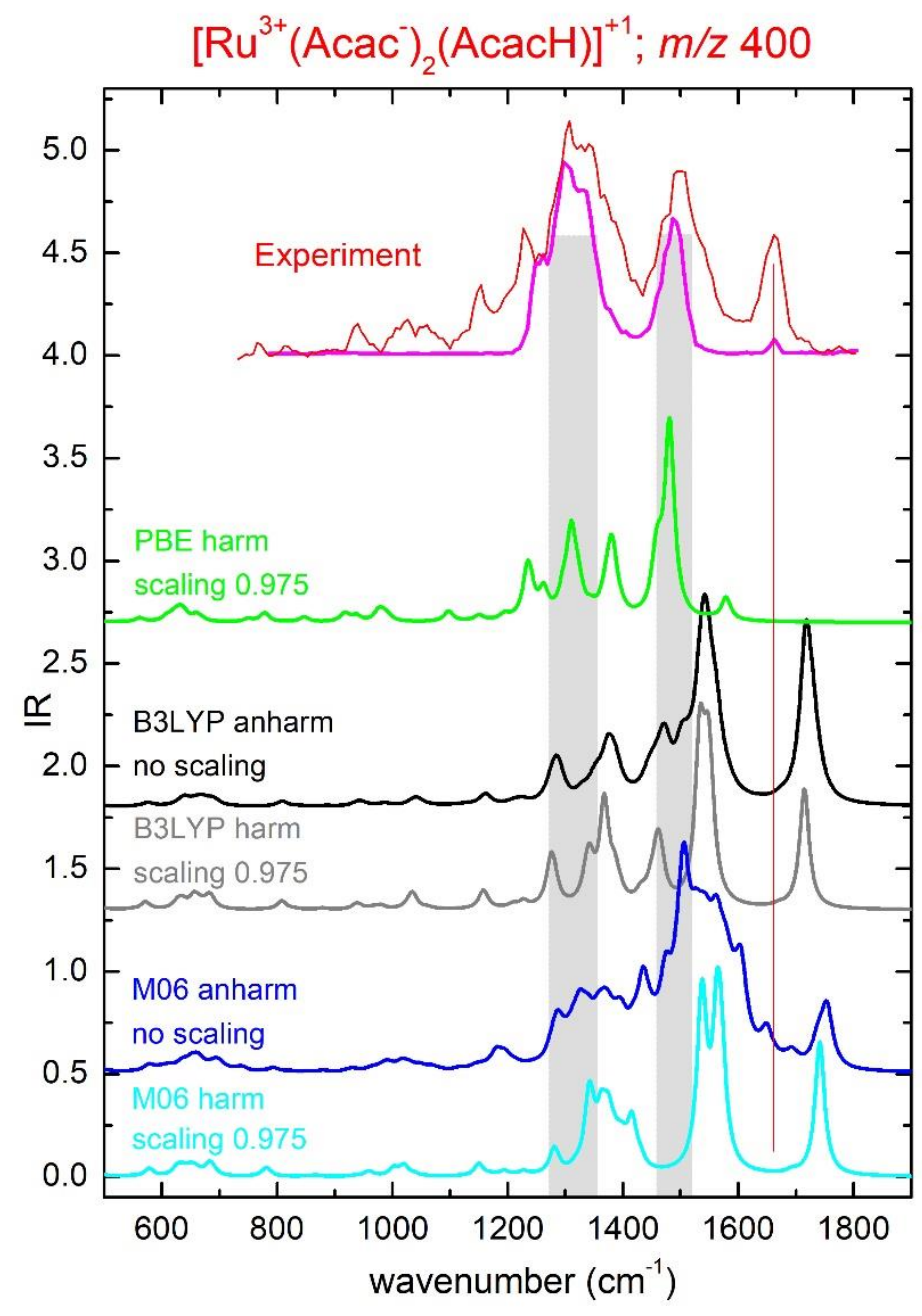

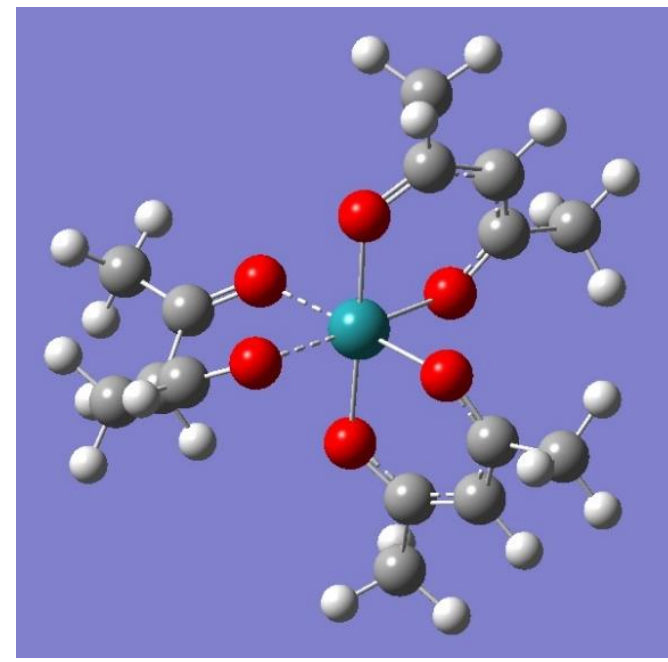

FIG. 3: same as fig. 2 for $\left[\mathrm{Ru}^{3+}\left(\mathrm{Acac}^{-}\right)_{2}(\mathrm{AcacH})\right]^{1+}$. The two experimental curves correspond to two runs in the same frequency range but with a $\mathrm{CO}_{2}$ laser pre-heating of ions (red) or not (magenta). Calculation results are displayed, using LanL2DZ basis set for Ru and either 6-31G* (B3LYP) or 6$31+G^{* *}(M 06)$ basis sets for all other atoms. For this complex, no PBE-DFT-MD calculation has been done but only scaled harmonic calculations. As for the same complexes with $\mathrm{Al}$ or $\mathrm{Fe}$, the resulting equilibrium structure displays a symmetric octahedral shape. 
As previously, the symmetric two $\mathrm{C}=\mathrm{O}$ stretches mode of neutral acetylacetone, here at $1665 \mathrm{~cm}^{-1}$, is still overestimated by at least $50 \mathrm{~cm}^{-1}$ in M06 and B3LYP calculations and largely underestimated, by at least $80 \mathrm{~cm}^{-1}$ in PBE calculations. The calculated equilibrium structure is displayed only for this $\left[\mathrm{Ru}^{3+}\left(\mathrm{Acac}^{-}\right)_{2}(\mathrm{AcacH})\right]^{1+}$ complex but its octahedral shape is also valid for $\mathrm{Al}$ and Fe complexes. The three complexes however differ by their M-O distances but also and more noticeably by their dissociation energies, as shown in Table I.

\begin{tabular}{|c|c|c|c|c|}
\hline $\mathrm{M}(\mathrm{III})$ ion & Spin & distance $\left({\mathrm{M}-\mathrm{O}^{-}}_{\text {Acac }}\right) \AA$ & distance $\left(\mathrm{M}^{-} \mathrm{O}_{\text {AcacH }}\right) \AA$ & $\mathrm{D}_{\mathrm{e}}(\mathrm{AcacH}) \mathrm{eV}$ \\
\hline $\mathrm{Al}$ & 0 & $1.85-1.87$ & $2.03-2.04$ & 1.50 \\
\hline $\mathrm{Fe}$ & $5 / 2$ & $1.93-1.99$ & $2.17-2.20$ & 1.63 \\
& $1 / 2$ & $1.85-1.89$ & $1.98-2.00$ & 2.45 \\
\hline $\mathrm{Ru}$ & $1 / 2$ & $1.97-2.04$ & $2.14-2.16$ & 2.38 \\
\hline
\end{tabular}

TABLE I: lower energy spin state, ranges of distances in $\AA$ between the metal ion and the oxygen atoms of either anionic Acac or neutral $\mathrm{AcacH}$ ligands and dissociation energy of this neutral ligand, for the three $\left[\mathrm{M}^{3+}\left(\text { Acac }^{-}\right)_{2}(\mathrm{AcacH})\right]^{1+}$ species, calculated at the $\mathrm{M} 06 / 6-31+\mathrm{G}^{* *}$ level. The second line (italic) for iron corresponds to $1 / 2$ spin state, which is of higher total electronic energy but with the same octahedral equilibrium structure and much stronger $\mathrm{M}-\mathrm{O}$ interactions and a much larger $\mathrm{AcacH}$ dissociation energy.

From these calculated data, it is clear that the $\mathrm{M}-\mathrm{O}$ distances and the $\mathrm{AcacH}$ dissociation energy depend on the size of the metal ion but are even more sensitive to the spin state of the complex. For iron, the ground electronic state is of high-spin $5 / 2$ (all 5 d-electrons are unpaired) but with lower M$\mathrm{O}$ interactions and a dissociation energy just slightly higher $(1.63 \mathrm{eV})$ than that of aluminum with no d-electron (1.50 eV). The higher energy low-spin state 1/2 (four d-electrons paired and only one $d$ electron unpaired) displays stronger $\mathrm{M}-\mathrm{O}$ interactions (shorter $\mathrm{d}(\mathrm{M}-\mathrm{O})$ distances) and a much higher dissociation energy $(2.45 \mathrm{eV})$, similar to that of the Ru complex (2.38 eV) whose ground state is the same low-spin state. We conclude that the large red-shift observed in the ruthenium IR spectrum, on several bands involving the electron donor centers of the ligands, in the $1200-1800 \mathrm{~cm}^{-1}$ range, can be used as a signature of strong metal-ligand interactions corresponding to a low-spin state of the complex.

\section{C. $\left[\mathrm{M}^{3+}\left(\mathrm{Acac}^{-}\right)_{2}\right]^{1+}, \mathrm{M}=\mathrm{Al}, \mathrm{Fe}$}

In those species, there are only two anionic bidentate ligands so that their structures are expected to be tetrahedral and strongly bound. Their fragmentation is indeed rather difficult and we never observed the doubly charged $\left[\mathrm{M}^{3+}\left(\mathrm{Acac}^{-}\right)_{1}\right]^{2+}$. Upon CID, the main fragments correspond to different neutral losses of the ligands while upon IRMPD we also observed a singly-charged fragment 
corresponding to the loss of one neutral acetylacetonate radical, while it does not appear upon CID.

Figure 4 displays the experimental IRMPD spectra for Al and Fe. Again, experimental data display the two or three runs that have been performed in different frequency ranges without (red) or with pre-heating (magenta and violet) so that spectral bands appear with various relative intensities that are normalized at their maximum.

Compared to previous species, spectra are simpler, as expected because of the higher symmetry due to the fact that all ligands are identical. The two main experimental bands are located around 1345 and $1520 \mathrm{~cm}^{-1}$ for $\mathrm{Al}$ and 1300 and $1490 \mathrm{~cm}^{-1}$ for Fe, i.e. $30-50 \mathrm{~cm}^{-1}$ red-shifted as compared to previous species which indicates stronger metal-ligand interactions in those two bidentate ligands complexes.
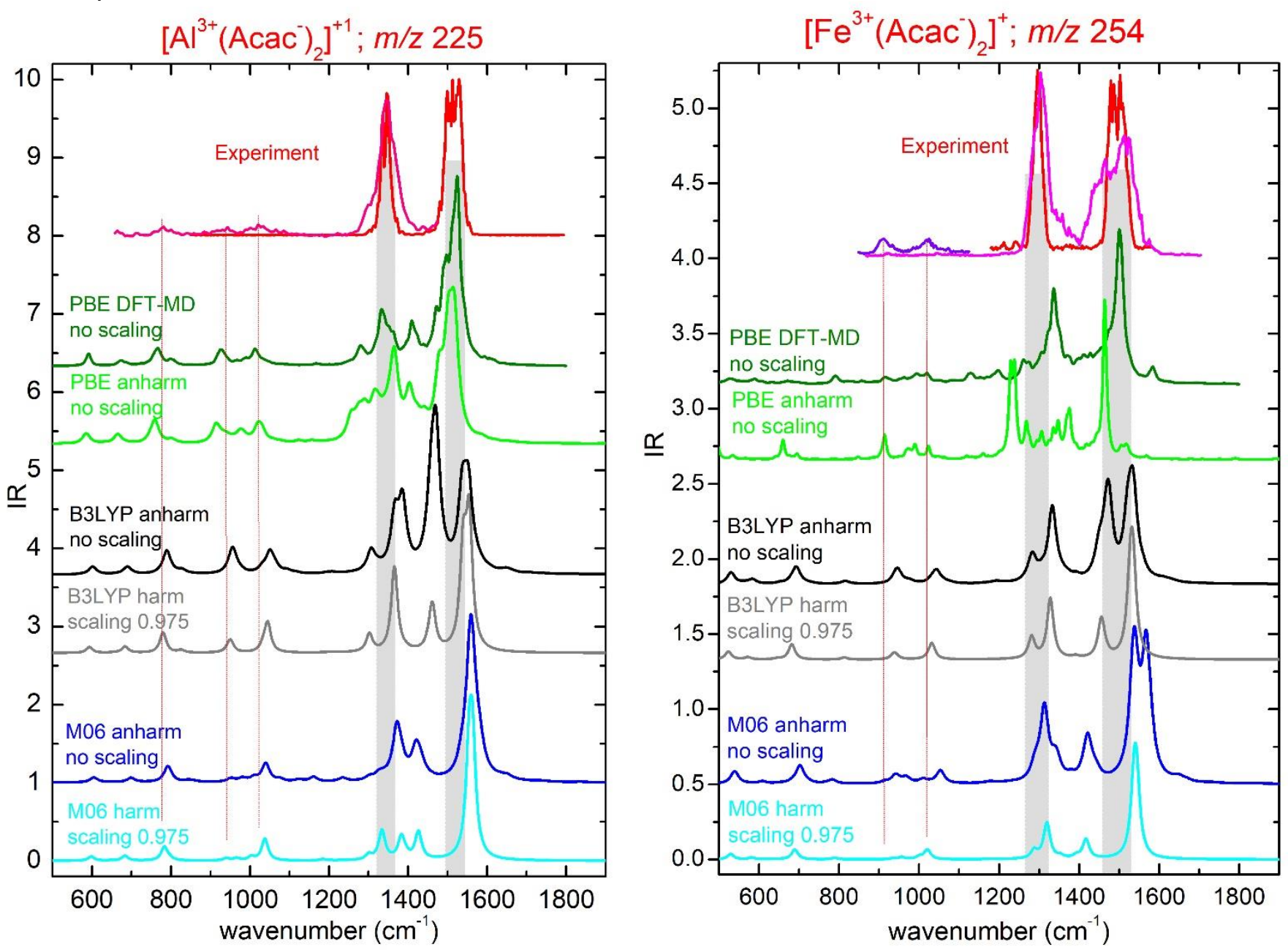

FIG. 4: experimental IRMPD spectra for $\left[\mathrm{Al}^{3+}\left(\mathrm{Acac}^{-}\right)_{2}\right]^{1+}$ and $\left[\mathrm{Fe}^{3+}\left(\mathrm{Acac}^{-}\right)_{2}\right]^{1+}$ (top curves) as compared to calculated scaled harmonic (light colors) and unscaled anharmonic (dark colors) IR spectra at the M06/6$31+G^{* *}$ (blue) and B3LYP/6-31G* (black), together with PBE/TZVP calculations performed at the unscaled anharmonic static level (light green) and at the unscaled dynamic level through DFT-MD simulations (dark green). The two main bands around 1300 and $1500 \mathrm{~cm}^{-1}$ are grayed out and few other lines are marked with dotted lines in order to guide eyes. 
Calculations reproduce quite well the positions of these two main bands, B3LYP performing again better than M06, but static calculations often display a double-peak shape for each experimental band. For those tetrahedral complexes, DFT-MD calculations much better reproduces the experimental spectra both for the band shapes, with only one large peak at the present frequency resolution, and for the positions, with no need of an empirical blue-shift correction as for previous complexes. Only few lines at lower frequencies are hardly detected, only when using $\mathrm{CO}_{2}$ laser preheating of trapped ions, most probably because the dissociative channels are here more endothermic. Again, whatever the theoretical method, calculations well reproduce the position of those lower frequency lines detected, with a slight blue-shift for B3LYP and M06. As expected, ground-state calculated structures (see Fig. 6 in the supplementary material) correspond to a tetrahedral geometry with the same $\mathrm{M}-\mathrm{O}$ distances for all four oxygen atoms, namely $1.78 \AA$ for $\mathrm{Al}$ and $1.88 \AA$ for Fe, i.e. shorter distances than in the previous octahedral complexes, in agreement with the stronger red-shifts observed.

\section{D. $\left[M^{3+}{ }_{2}\left(\mathrm{Acac}^{-}\right)_{5}\right]^{1+}, \mathrm{M}=\mathrm{Al}, \mathrm{Fe}$}

In those species, the two metal ions can recover an octahedral environment if one or two bidentate ligands make a bridge between the two cationic centers so that they can share two anionic centers. Their fragmentation by CID or IRMPD is rather easy and occurs mainly through the loss of the very stable $\mathrm{M}^{3+}\left(\mathrm{Acac}^{-}\right)_{3}$ neutral fragments. Figure 5 displays the corresponding experimental IRMPD spectra for $\mathrm{Al}$ and $\mathrm{Fe}$, with two runs that have been performed in the low- and high-frequency ranges.

The two spectra display several similarities in shape and position with those of the mono-metallic $\left[\mathrm{M}^{3+}(\text { Acac- })_{2}(\mathrm{AcacH})\right]^{1+}$ complexes (Fig. 2), as expected if they share the same structure symmetry. The two main experimental bands are located around 1400 and $1560 \mathrm{~cm}^{-1}$ for Al and 1380 and 1580 $\mathrm{cm}^{-1}$ for Fe, i.e. close to the same bands in the mono-metallic ions, with the same small blue-shift of $+7 \mathrm{~cm}^{-1}$ for $\mathrm{Al}$ and $+24 \mathrm{~cm}^{-1}$ for Fe (see Table III in the supplementary material). This is a further indication that they should share the same structure and spin state but with some additional structure constraints in the case of bi-metallic complexes that lead to slightly weaker metal-ligand interactions and thus slightly higher frequencies for those modes involving vibrations of the ligand backbone that are slightly less affected by metal ion binding. This interpretation also holds for the lower frequency modes, below $1100 \mathrm{~cm}^{-1}$, which are also similar but with small red-shifts in that case $\left(0\right.$ to $\left.-20 \mathrm{~cm}^{-1}\right)$, 
since they correspond mainly to metal-ligand vibrations that are weaker in the bi-metallic complexes (see Table III, modes 1 to 4 , in the supplementary material).

However, we were able to detect one more specific low-frequency band, at $526 \mathrm{~cm}^{-1}$ for Al (very weak) and $447 \mathrm{~cm}^{-1}$ for Fe (stronger), which also appears in the calculations for the lowest double octahedron structure and corresponds to several modes of similar frequencies but all involving the ligands that are shared by the two metal ion centers. The main difference with the mono-metallic complexes comes from the high-energy line $\left(1730 \mathrm{~cm}^{-1}\right)$ that is here absent, obviously because there is no neutral ligand.
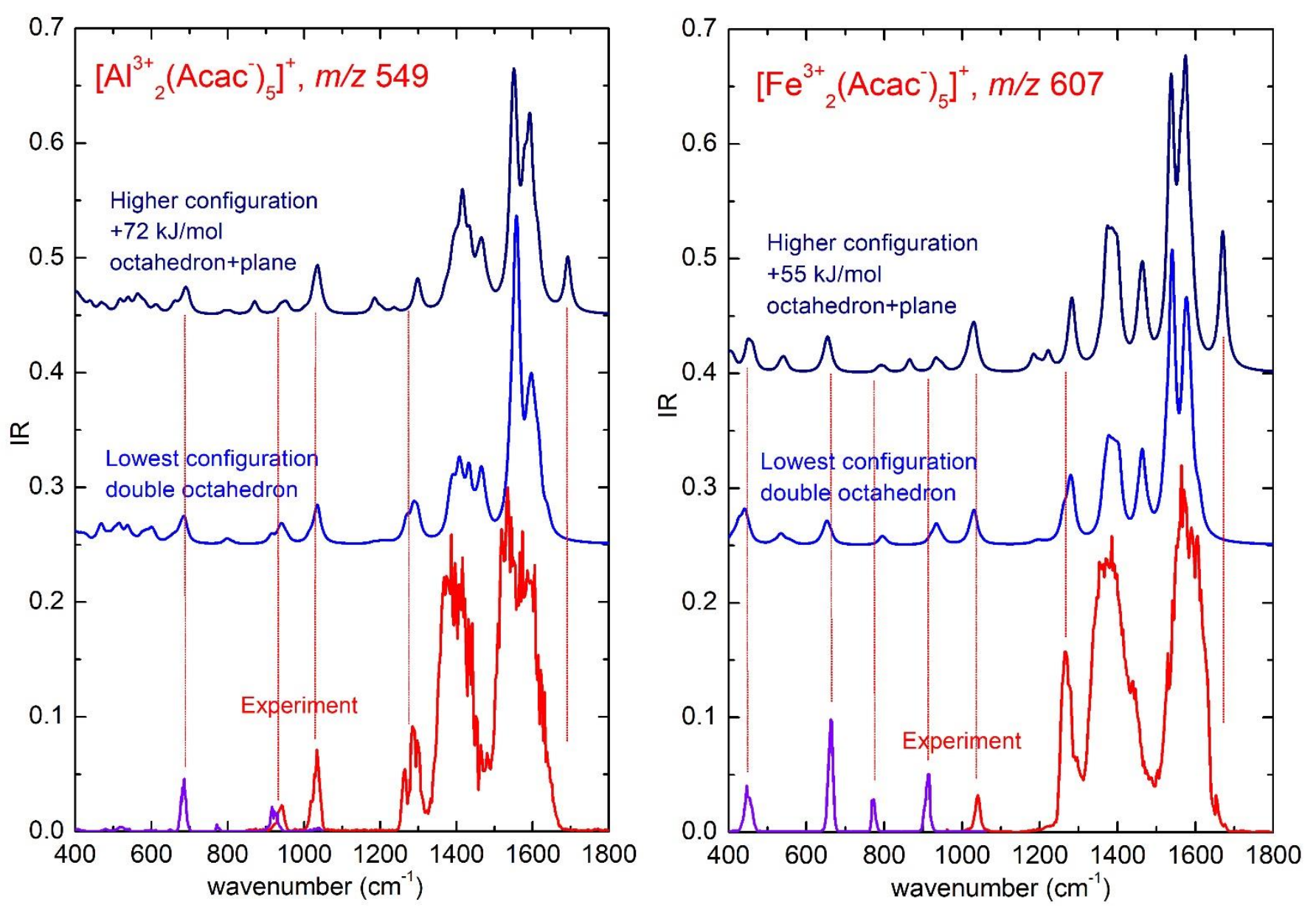

FIG. 5: experimental IRMPD spectra for $\left[\mathrm{Al}^{3+}{ }_{2}\left(\mathrm{Acac}^{-}\right)_{5}\right]^{1+}$ and $\left[\mathrm{Fe}^{3+}{ }_{2}\left(\mathrm{Acac}^{-}\right)_{5}\right]^{1+}$ (bottom curves) as compared to calculated scaled harmonic IR spectra at the B3LYP/6-31G* level, for the lowest energy structure (double octahedron) and for the higher energy configuration (octahedron+plane). Note that the frequency scaling factor was not adjusted but conserved at its previous value of 0.975 and that ferromagnetic coupling was assumed between metal centers.

Finally, we have tested one higher energy structure which consists of a mono-metallic octahedral structure to which a more or less planar $\left[\mathrm{M}^{3+}\left(\mathrm{Acac}^{-}\right)_{2}\right]^{1+}$ species is bound mainly through an interaction between this second metal ion and the $\mathrm{C}_{3}$ carbon atom of an Acac ligand doubly bound to the first metal ion. 
This structure is displayed in Fig. 6, together with the double octahedral structure, but it lies at much higher energy, $+72 \mathrm{~kJ} / \mathrm{mol}$ for $\mathrm{Al}$ and $+55 \mathrm{~kJ} / \mathrm{mol}$ for Fe. Moreover, the corresponding calculated spectra exhibit a line at $1690 \mathrm{~cm}^{-1}(\mathrm{Al})$ or $1670 \mathrm{~cm}^{-1}(\mathrm{Fe})$, corresponding to the symmetric $\mathrm{C}=\mathrm{O}$ stretches of the ligand which $\mathrm{C}_{3}$ atom interacts with one metal ion. Since these lines are not experimentally observed, we rule out the presence of this second conformer in our experiments.

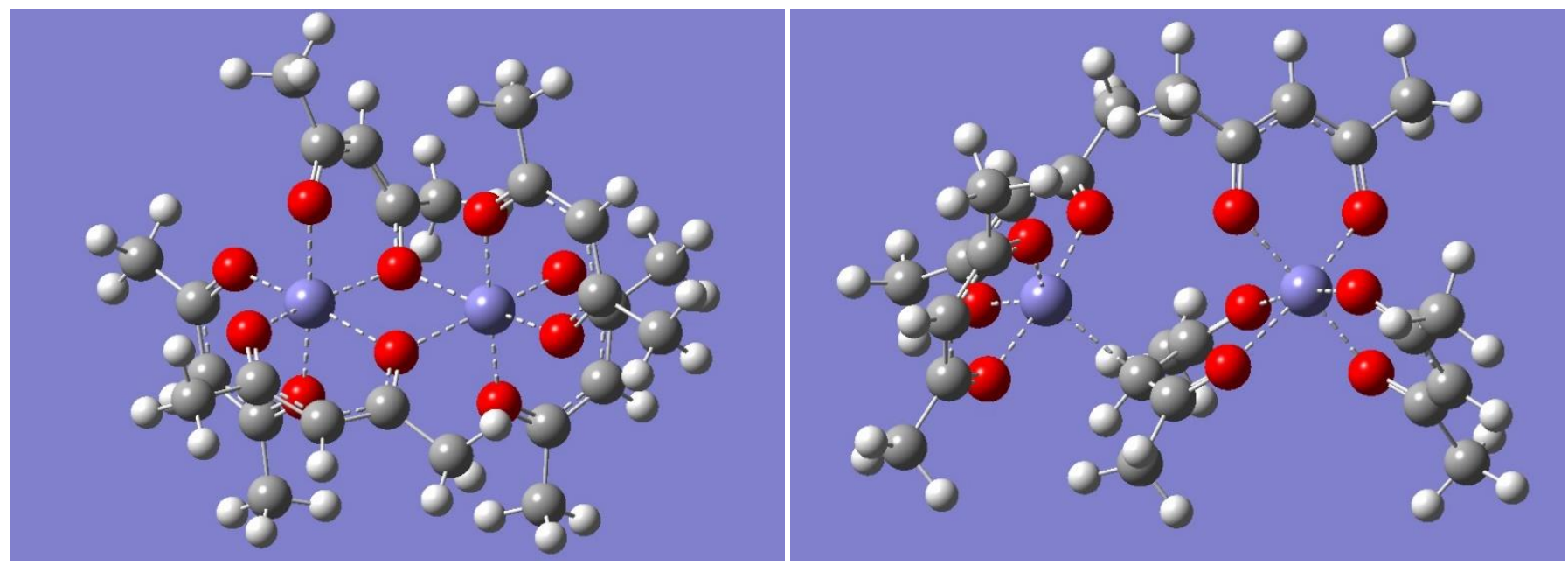

FIG. 6: two equilibrium structures for $\left[\mathrm{Fe}^{3+}{ }_{2}\left(\mathrm{Acac}^{-}\right)_{5}\right]^{1+}$, as calculated at the B3LYP/6-31G* level, for the lowest energy conformer (left: double octahedron) and for the higher energy configuration (right: octahedron+plane); in this conformer, there is a specific interaction between an iron ion and $\mathrm{a}_{3}$ carbon atom of one anionic ligand (dash line in the center), but the total energy is much higher than that of the double octahedron structure.

Finally for Al (as displayed in Fig. 7 of the supplementary material), we were able to obtain the IRMPD spectra of a species at $m / z 467$ which corresponds either to $\left[\mathrm{Al}^{3+}{ }_{2}\left(\mathrm{Acac}^{-}\right)_{4} \mathrm{OH}^{-}\right]^{1+}$ or to $\left[\mathrm{Al}^{3+}{ }_{2}\left(\mathrm{Acac}^{-}\right.\right.$ )$\left._{3}(\mathrm{AcacH}) \mathrm{O}^{2-}\right]^{1+}$, i.e. the same complex in which one Acac ${ }^{-}$ion has been replaced by an hydroxyl $\mathrm{OH}^{-}$ anion or two $\mathrm{Acac}^{-}$ions by one neutral $\mathrm{AcacH}$ plus a superoxide $\mathrm{O}^{2-}$ anion. From calculations, both isomers are of similar distorted octahedral geometry but the second isomer is located at much higher energy $(+172 \mathrm{~kJ} / \mathrm{mol})$. In agreement with these calculations, the experimental spectrum fits well to the calculated one only for the first hydroxyl-containing isomer (presence of a line at $920 \mathrm{~cm}^{-1}$ and absence at $\left.1785 \mathrm{~cm}^{-1}\right)$. 


\section{CONCLUSION}

In this work, we have performed IRMPD spectroscopy experiments on strongly bound $\mathrm{M}^{3+}$-Acaccomplexes with different octahedral or tetrahedral structures. We have shown that the spectral shift of the vibrational modes relative to the $\mathrm{O}=\mathrm{C}-\mathrm{C}-\mathrm{C}=\mathrm{O}$ part of the Acac ligand, in the frequency range in between 1200 and $1700 \mathrm{~cm}^{-1}$, is directly related to the strength of the $\mathrm{M}^{3+}-\mathrm{O}$ interactions and to the spin state. The cases of $\left[\mathrm{M}^{3+}\left(\mathrm{Acac}^{-}\right)_{2}(\mathrm{AcacH})\right]^{1+}$ and $\left[\mathrm{M}^{3+}{ }_{2}\left(\mathrm{Acac}^{-}\right)_{5}\right]^{1+}$ ions are particularly interesting because they can fragment relatively more easily and lead to rich IR spectra even for lowfrequency $\left(400-1000 \mathrm{~cm}^{-1}\right)$ low-intensity modes that are characteristic of the ligand structure and, for the lowest frequency ones, of the $\mathrm{M}^{3+}-\mathrm{O}$ interactions too.

Concerning IR spectra simulations, these species are good benchmark systems to compare with different theoretical approaches. In this study, we used DFT calculations with three different functionals (B3LYP, M06 and PBE) and three different spectra calculation (scaled harmonic, static anharmonic and MD simulations). All methods lead to simulated spectra that fit rather well with experimental data, although none of them seems to be always quantitative for the higher fingerprint frequency range (above $1200 \mathrm{~cm}^{-1}$ ), even for the DFT-MD simulations, while the agreement is much better for the low-frequency range (below $1000 \mathrm{~cm}^{-1}$ ). From this observation, we can conclude that, due to the rigidity of the complexes, the spectra calculated using first principles molecular dynamics does not provide a different picture with respect to the static ones. Even a simple and low-cost method, such as scaled static harmonic calculations at the B3LYP/6-31G* level, is already enough to describe the main IR vibrational features that are experimentally observed with the right structure and the right spin state. This conclusion is in good agreement with previous compared IRMPD/theoretical studies [54].

This study on simple species allows us to build some methodology benchmarks for further spectroscopic experiments and DFT calculations focused on larger model systems such as real liganded nanoparticles, as used by biochemists. Indeed, several other experiments are underway on more complex ligands and complexes of larger size, in order to further test the above provisional conclusions and to progress towards systems that are more relevant to characterize the physicochemical properties of small nanoparticles. 


\section{SUPPLEMENTARY MATERIAL}

Supplementary material includes full mass spectra of the samples studied together with few isotopic patterns of interest, several tables of experimental and calculated vibrational modes for octahedral mono- and bi-metallic complexes and experimental and calculated IRMPD spectra for species at $\mathrm{m} / \mathrm{z}$ 467 corresponding to oxidized bi-metallic ions.

\section{DATA AVAILABILITY}

The data that support the findings of this study are available from the corresponding author upon reasonable request.

\section{ACKNOWLEDGMENTS}

We are grateful to Cineca and PRACE for computational support through grants IsC71_AIMDAcAc and Pra16_3574 and to the MAGI HPC computing center and its engineer Nicolas Grenèche.

We also thank the CLIO facility and its technical staff, in particular Estelle Loire, Fabrice Gobert and Nicolas Jestin for their efficient assistance. The French FT-ICR network (CNRS, FR 3624) is also acknowledged for the access to the MS setup.

Finally, we are also grateful to Jeanne Crassous (Rennes Institute of Chemical Sciences), for providing us a sample of $\mathrm{Ru}(\mathrm{Acac})_{3}$. 


\section{REFERENCES}

[1] M.B. Cortie, A.M. McDonagh, Synthesis and optical properties of hybrid and alloy plasmonic nanoparticles, Chem. Rev. 111, 3713 (2011).

[2] M. Turner, V.B. Golovko, O.P. Vaughan, P. Abdulkin, A. Berenguer-Murci, M.S. Tikhov, B.F. Johnson, R.M. Lambert, Selective oxidation with dioxygen by gold nanoparticle catalysts derived from 55-atom clusters, Nature 454, 981 (2008).

[3] C.-A.J. Lin, C.-H. Lee, J.-T. Hsieh, H.-H. Wang, J.K. Li, J.-L. Shen, W.-H. Chan, H.-I. Yeh, W.H. Chang, Synthesis of fluorescent metallic nanoclusters toward biomedical application: recent progress and present challenges, J. Med. Biol. Eng. 29, 276 (2009).

[4] J.H. Jung, J.H. Lee, S. Shinkai, Functionalized magnetic nanoparticles as chemosensors and adsorbents for toxic metal ions in environmental and biological fields, Chem. Soc. Rev. 40, 4464 (2011).

[5] C.M. Cobley, J. Chen, E.C. Cho, L.V. Wang, Y. Xia, Gold nanostructures: a class of multifunctional materials for biomedical applications, Chem. Soc. Rev. 40, 44 (2011).

[6] B. Zhang, B. Yan, Analytical strategies for characterizing the surface chemistry of nanoparticles, Anal. Bioanal. Chem. 396, 973 (2010).

[7] Y. Lu, W. Chen, Sub-nanometer sized metal clusters: from synthetic challenges to the unique property discoveries, Chem. Soc. Rev. 41, 3594 (2012).

[8] Li Shang, S. Dong, G.U. Nienhaus, Ultra-small fluorescent metal nanoclusters: Synthesis and biological applications, Nano Today 6, 401 (2011).

[9] L. MacAleese, P. Maitre, Infrared Spectroscopy of Organometallic lons in the Gas Phase: from Model to Real World Complexes, Mass Spectrom. Rev. 26, 583 (2007) and references therein.

[10] J. Oomens, N.C. Polfer, G. Berden, J.R. Eyler, Gas-phase metal ion chelation investigated with IRMPD spectroscopy: A brief review of Robert Dunbar's contributions, Eur. J. Mass Spectrom. 25, 86 (2019) and references therein.

[11] M.A. Duncan, Infrared spectroscopy to probe structure and dynamics in metal ion-molecule complexes, Int. Reviews in Physical Chemistry 22, 407 (2003).

[12] P. Sharpe, D.E. Richardson, Metal-ligand bond energies and solvation energies for gas-phase transition-metal tris (acetylacetonate) complexes and their negative ions, J. Am. Chem. Soc. 113, 8339 (1991).

[13] S. Hoyau, K. Norrman, T. B. McMahon, G. Ohanessian, A Quantitative Basis for a Scale of $\mathrm{Na}+$ Affinities of Organic and Small Biological Molecules in the Gas Phase, J. Am. Chem. Soc.

121, 8864 (1999). 
[14] S. Le Caer, M. Heninger, J. Lemaire, P. Boissel, P. Maitre, H. Mestdagh, Structural characterization of selectively prepared cationic iron complexes bearing monodentate and bidentate ether ligands using infrared photodissociation spectroscopy, Chem. Phys. Lett. 385, 273 (2004).

[15] D.J. Miller, J.M. Lisy, Entropic Effects on Hydrated Alkali-Metal Cations: Infrared Spectroscopy and ab Initio Calculations of $M+(H 2 O) x=2-5$ Cluster lons for $M=L i, N a, K$, and Cs, J. Am. Chem. Soc. 130, 15393 (2008).

[16] A. Fielicke, G. von Helden, G. Meijer, B. Simard, S. Dénommé, D.M. Rayner, Vibrational Spectroscopy of CO in Gas-Phase Rhodium Cluster-CO Complexes, J. Am Chem. Soc. 125, 11184 (2003).

[17] M.J. van Stipdonk, P. Basu, S.A. Dille, J.K. Gibson, G. Berden, J. Oomens, Infrared Multiple Photon Dissociation Spectroscopy of a Gas-Phase Oxo-Molybdenum Complex with 1,2-Dithiolene Ligands, J. Phys. Chem. A 118, 5407 (2014).

[18] N.C. Polfer, J. Oomens, D.T. Moore, G. von Helden, G. Meijer, R.C. Dunbar, Infrared Spectroscopy of Phenylalanine Ag(I) and Zn(II) Complexes in the Gas Phase, J. Am. Chem. Soc. 128, 517 (2006).

[19] R. Otsuka, K. Hirata, Y. Sasaki, J.M. Lisy, S.Ishiuchi, M. Fujii, Alkali and alkaline earth metal ions complexes with a partial peptide of the selectivity filter in $K+$ channels studied by a cold ion trap infrared spectroscopy, ChemPhysChem 21, 712 (2020).

[20] C. Kapota, J. Lemaire, P. Maitre, G. Ohanessian, Vibrational Signature of Charge Solvation vs Salt Bridge Isomers of Sodiated Amino Acids in the Gas Phase, J. Am. Chem. Soc. 126, 1836 (2004).

[21] G.C. Boles, C.J. Owen, G. Berden, J. Oomens, P. B. Armentrout, Experimental and theoretical investigations of infrared multiple photon dissociation spectra of glutamic acid complexes with Zn2+ and Cd2+, Phys. Chem. Chem. Phys. 19, 12394 (2017).

[22] A. Günther, P. Nieto, G. Berden, J. Oomens, O. Dopfer, IRMPD spectroscopy of metalated flavins: structure and bonding of $\mathrm{Mq}+$-lumichrome complexes (Mq+ $\left.=\mathrm{Li}+\mathrm{Cs}+, \mathrm{Ag}+, \mathrm{Mg} \mathrm{L}_{+}\right)$, Phys. Chem. Chem. Phys. 16, 14161 (2014).

[23] K.J. Houthuijs, J. Martens, A.G. Arranja, G. Berden, J.F.W. Nijsen, J. Oomens, Characterization of holmium(III)-acetylacetonate complexes derived from therapeutic microspheres by infrared ion spectroscopy, Phys.Chem.Chem.Phys. 22, 15716 (2020).

[24] I. Diaz-Acosta, J. Baker, W. Cordes, P. Pulay, Calculated and Experimental Geometries and Infrared Spectra of Metal Tris-Acetylacetonates: Vibrational Spectroscopy as a Probe of Molecular Structure for lonic Complexes. Part I, J. Phys. Chem. A 105, 238 (2001). 
[25] P.A. Stabnikov, N.V. Pervukhina, I.A. Baidina, L.A. Sheludyakova, S.V. Borisov, On the Symmetry of Iron(III)tris-acetylacetonate Crystals, J. Struct. Chem. 48, 186 (2007) and references therein.

[26] S.N. Slabzhennikov, O.B. Ryabchenko, L.A. Kuarton, Normal Vibration Calculations for Iron Tris(acetylacetonate), Russian J. Coord. Chem. 29, 484 (2003) and references therein.

[27] P. Guardia, N. Perez, A. Labarta, X. Batlle, Controlled Synthesis of Iron Oxide Nanoparticles over a Wide Size Range, Langmuir 26, 5843 (2010) and references therein.

[28] L. Jasikova, J. Roithova, Infrared Multiphoton Dissociation Spectroscopy with Free-Electron Lasers: from Small Molecules to Biomolecules, Chem. Eur. J. 24, 3374 (2018) and references therein.

[29] R.L. Woodin, D.S. Bomse, J.L. Beauchamp, Multi-photon dissociation of molecules with lowpower continuous wave infrared-laser radiation, J. Am. Chem. Soc. 100, 3248 (1978).

[30] P. Maitre, D. Scuderi, D. Corinti, B. Chiavarino, M.E. Crestoni, S. Fornarini, Applications of infrared multiple photon dissociation (IRMPD) to the detection of posttranslational modifications, Chem. Rev. 120, 3261 (2020).

[31] R. Prazeres, F. Glotin, C. Insa, D.A. Jaroszynski, J.M. Ortega, Two-color operation of a freeelectron laser and applications in the mid-infrared, Eur. Phys. J. D 3, 87 (1998).

[32] J.M. Bakker, T. Besson, J. Lemaire, D. Scuderi, P. Maitre, Gas-phase structure of a $\pi$-AllylPalladium complex: efficient infrared spectroscopy in a 7 T Fourier transform mass spectrometer, J. Phys. Chem. A 111, 13415 (2007).

[33] D.J. Nesbitt, R.W. Field, Vibrational energy flow in highly excited molecules: Role of intramolecular vibrational redistribution, J. Phys. Chem. 100, 12735 (1996).

[34] Gaussian 16, Revision C.01, M.J. Frisch, Gaussian, Inc., Wallingford CT (2016).

[35] A. D. Becke, A new mixing of Hartree-Fock and local density functional theories, J. Chem. Phys. 98,1372 (1993).

[36] C. Lee, W. Yang, R. G. Parr, Development of the Colle-Salvetti correlation-energy formula into a functional of the electron density, Phys. Rev. B 37, 785 (1988).

[37] S. H. Vosko, L. Wilk, M. Nusair, Accurate spin-dependent electron liquid correlation energies for local spin density calculations: a critical analysis, Can. J. Phys. 58, 1200 (1980).

[38] R. H. W. J. Ditchfield, W. J. Hehre, J. A. Pople, Self- consistent molecular- orbital methods. IX. An extended Gaussian-type basis for molecular orbital studies of organic molecules, J. Chem. Phys. 54, 724 (1971). 
[39] F. Weigend, R. Ahlrichs, Balanced basis sets of split valence, triple zeta valence and quadruple zeta valence quality for $H$ to $R n$ : Design and assessment of accuracy, Phys. Chem. Chem. Phys. 7, 3297 (2005).

[40] P.J. Hay, W.R. Wadt, Ab initio effective core potentials for molecular calculations. Potentials for the transition metal atoms Sc to Hg, J. Chem. Phys. 82, 270 (1985).

[41] Y. Zhao, D.G. Truhlar, The M06 suite of density functionals for main group thermochemistry, thermochemical kinetics, noncovalent interactions, excited states, and transition elements: two new functionals and systematic testing of four M06-class functionals and 12 other functionals, Theo. Chem. Acc. 120, 215 (2008).

[42] C.J. Cramer, D.G. Truhlar, Density functional theory for transition metals and transition metal chemistry, Phys. Chem. Chem. Phys. 11, 10757 (2009).

[43] L.M.L. Daku, F. Aquilante, T.W. Robinson, A. Hauser, Accurate spin-state energetics of transition metal complexes.1. $\operatorname{CCSD}(T), \mathrm{CASPT2}$, and DFT study of $\left[\mathrm{M}(\mathrm{NCH})_{6}\right]^{2+}(\mathrm{M}=\mathrm{Fe}, \mathrm{Co})$, J. Chem. Theory Comput. 8, 4216 (2012).

[44] C. Møller, M. S. Plesset, Note on an approximation treatment for many-electron systems, Phys. Rev. 46, 618 (1934).

[45] J. Hutter, M. Iannuzzi, F. Schiffmann, J. VandeVondele, cp2k: atomistic simulations of condensed matter systems, WIREs Comput. Mol. Sci. 4, 15 (2014).

[46] N. Marzari, D. Vanderbilt, Maximally localized generalized Wannier functions for composite energy bands, Phys. Rev. B 56, 2847 (1997).

[47] J. P. Perdew, K. Burke, M. Ernzerhof, Generalized gradient approximation made simple, Phys. Rev. Lett. 77, 3865 (1996).

[48] A. Schäfer, H. H Horn, R. Ahlrichs, Fully optimized contracted Gaussian basis sets for atoms Li to Kr, J. Chem. Phys. 97, 2571 (1992).

[49] S. Goedecker, M. Teter, and J. Hutter, Separable dual-space Gaussian pseudopotentials, Phys. Rev. B 54, 1703 (1996).

[50] S. Grimme, J. Antony, S. Ehrlich, H. Krieg, A consistent and accurate ab initio parametrization of density functional dispersion correction (DFT-D) for the 94 elements $\mathrm{H}-\mathrm{Pu}$, J. Chem. Phys. 132 , 154104 (2010).

[51] P.K. Hon, C.E. Pfluger, The Crystal and Molecular Structure of tris(acetylacetonato)Aluminum(III) and-Cobalt(III), J. Coord. Chem. 3, 67 (1973).

[52] G.K-J. Chao, R.L. Sime, R.J. Sime, The Crystal and Molecular Structure of Trisacetylacetonatoruthenium(III), Acta Cryst. 29, 2845 (1973). 
[53] S.P. Sousa, P.A. Fernandes, M.J. Ramos, General performance of density functionals, J. Phys. Chem. A 111, 10439 (2007).

[54] M. Katari, E. Nicol, V. Steinmetz, G. van der Rest, D. Carmichael, G. Frison, Improved Infrared Spectra Prediction by DFT from a New Experimental Database, Chem. Eur. J. 23, 8414 (2017). 